\title{
Monotone recurrence relations, their Birkhoff orbits and topological entropy
}

\author{
SIGURD B. ANGENENT $\dagger$ \\ University of Wisconsin-Madison Center for the Mathematical Sciences, \\ Wisconsin, USA
}

(Received 1 March 1988)

\begin{abstract}
A generalization of the class of monotone twistmaps to maps of $S^{1} \times R^{N}$ is proposed. The existence of Birkhoff orbits is studied, and a criterion for positive topological entropy is given. These results are then specialized to the case of monotone twist maps. Finally it is shown that there is a large class of symplectic maps to which the foregoing discussion applies.
\end{abstract}

\section{Introduction}

In this paper we shall study a class of dynamical systems which generalizes the class of 'monotone twist maps' of two dimensional annuli, and also the class of 'degree one circle maps', both of which have been studied extensively in the last decade or two.

The maps which we shall deal with are continuous maps $\varphi$ of the generalized annulus $S^{1} \times R^{N}(N \geq 0)$ into itself. The defining condition of these maps is that they come from solving a recurrence relation

$$
\Delta\left(x_{k-l}, \ldots, x_{k+m}\right)=0 \quad(\forall k \in Z)
$$

in the first coordinate of a general point in $S^{1} \times R^{N}$. This is explained more precisely in $\S 2$.

Our main concern is with the construction of Birkhoff orbits of $\varphi$ (defined in $\S 3$ ), and a criterion for positivity of the topological entropy, $h_{\text {top }}(\varphi)$, of the map $\varphi$.

The main tool which we use is presented in $\$ 4$, in the form of Theorem 4.2 and two afterthoughts. This theorem allows us to construct solutions of the recurrence equation with prescribed qualitative properties. The method of construction is a discrete analog of Perron's method of solving the Dirichlet problem i.e. his method of constructing harmonic functions with prescribed boundary values from subharmonic functions and 'barrier functions' (or super harmonic functions).

As a first application of this method we show in $\$ 5$ how the existence of certain kinds of orbits of $\varphi$ implies the existence of a Birkhoff orbit, thereby generalizing a result of Hall ([Ha1]).

† This research was partially supported by the United States Army under Contract No. DAAL03-87-K0043 , by a NATO Science Fellowship, and by the Netherlands Organization for the Advancement of Pure Research (Z.W.O.). 
In $\S 6$ we formulate Hypothesis 6.1 , which roughly speaking, asserts the existence of two solutions of (1.1), say $x_{n}^{(1)}$ and $x_{n}^{(2)}$, which 'exchange rotation numbers' in the sense that

$$
\lim _{n \rightarrow \infty} \frac{x_{n}^{(1)}}{n} \geq \omega_{1} \geq \lim _{n \rightarrow-\infty} \frac{x_{n}^{(2)}}{n}
$$

and

$$
\lim _{n \rightarrow \infty} \frac{x_{n}^{(2)}}{n} \leq \omega_{0} \leq \lim _{n \rightarrow-\infty} \frac{x_{n}^{(1)}}{n}
$$

hold for some $\omega_{0}<\omega_{1}$. It is shown that this hypothesis implies the existence of Birkhoff orbits with any prescribed rotation number $\omega$ in the interval $\left[\omega_{0}, \omega_{1}\right]$.

A more complicated construction, which is carried out in $\S 7$, shows that the Hypothesis (6.1) implies that $h_{\text {top }}(\varphi)>0$. In fact we show that there is a compact set $K \subset S^{1} \times R^{N}$ such that some iterate of $\varphi$ leaves $K$ invariant, and has a Bernoulli shift as a factor, when restricted to $K$. Here we partially generalize another result of Hall, who in turn was concerned with giving a topological version of a 'shadowing theorem' of Mather's (see [Ha2] and [Ma2] where Mather's result is announced). A consequence of this construction is that the number of periodic orbits with period $\leq N$ grows exponentially with $N$. Moreover, our method of proving existence of these orbits is constructive. Given $x^{(1)}$ and $x^{(2)}$ a computer program could be written which constructs a great number of periodic orbits.

In $\$ 8$ we specialize our results to the two dimensional case, and ask which properties a map (i.e. a monotone twist map) with zero topological entropy must have. The first result in this direction is that, if $h_{\text {top }}(\varphi)=0$, then any orbit must have a forward and backward rotation number. Similar results, under different hypotheses, have been obtained by M. Handel. The other result is that, if $h_{\text {top }}(\varphi)=0$, then any periodic orbit 'of type $(p, q)$ ' with $\operatorname{gcd}(p, q)=1$ must be a Birkhoff orbit. This result is originally due to Boyland [Bo]. As far as I am aware, both Handel and Boyland rely on Thurston's classification of surface diffeomorphisms. By contrast, our approach is self contained, and as we have pointed out before, constructive, in a certain sense.

Throughout $\S 8$ we could have weakened the hypothesis ' $h_{\text {top }}(\varphi)=0$ ' to 'there is no invariant set $K \subset S^{1} \times R^{1}$ such that $\varphi \mid K$ has a subshift of finite type as a factor'. However, a theorem of Katok ([Ka2]) shows that this is not very much of a weakening. Indeed, if the map $\varphi$ is $C^{1+F}$, then his theorem says that both conditions are equivalent.

The next section, the ninth, deals with the question of existence of Birkhoff orbits in general, i.e. without further hypotheses on the map $\varphi$. We obtain the existence of Birkhoff orbits with prescribed rotation number $\omega$, not for the original map $\varphi$, but for a 'translated map' which comes from the recurrence relation $\Delta\left(x_{k-l}, \ldots, x_{k+m}\right)=\lambda$ for some $\lambda$. In general this $\lambda$ will depend on $\omega$, and will not be zero. From this result we derive a sufficient condition of the existence of at least one Birkhoff orbit of the original map $\varphi$. The condition turns out to be (trivially) necessary as well. We conclude this section by briefly specializing the results to the 
one dimensional (i.e. degree-one circle maps) and the two dimensional (twist map) case.

Finally, in the last section, by way of example, we indicate a number of symplectic maps $\varphi$ which belong to the class of maps which was studied in this paper. In particular we show that there is a very simple mechanical model, whose steady states are described by such maps.

\section{Twist maps and recurrence relations}

Let $A$ be the cylinder $(\mathbf{R} / \mathbf{Z}) \times \mathbf{R}$, and let $\varphi: A \rightarrow A$ be an orientation preserving monotone twist homeomorphism. On the universal cover $\mathbf{R}^{2}$ of $A$ a lift of $\varphi$ will be given by

$$
F(x, y)=(f(x, y), g(x, y)),
$$

where $f, g$ are periodic in the sense that

$$
\begin{aligned}
& f(x+1, y)=f(x, y)+1 \\
& g(x+1, y)=g(x, y)
\end{aligned}
$$

(i.e. $x$ is the angle coordinate). The monotone twist condition on $\varphi$ says that the function $f(x, y)$ is strictly increasing in $y$.

In addition we shall assume that $\varphi$ satisfies the infinite twist condition:

$$
\lim _{y \rightarrow \pm \infty} f(x, y)= \pm \infty \text {. }
$$

Combined, these two conditions imply that for any pair $x, \bar{x} \in \mathbf{R}$ there is a unique solution $Y(x, \bar{x})$ of the equation

$$
f(x, Y(x, \bar{x}))=\bar{x} .
$$

This solution is a strictly increasing function of $\bar{x}$. It is continuous, and satisfies

$$
Y(x, \bar{x})=Y(x+1, \bar{x}+1)
$$

for all $x, \bar{x} \in \mathbf{R}$.

From $Y(x, \bar{x})$ we construct another function:

$$
\bar{Y}(x, \bar{x})=g(x, Y(x, \bar{x})) .
$$

This function is also continuous, and periodic in the sense that

$$
\bar{Y}(x, \bar{x}) \equiv \bar{Y}(x+1, \bar{x}+1) .
$$

We shall now show that $Y(x, \bar{x})$ is a strictly decreasing functon of $x$.

Fox fixed $x_{0}$ the image of the line $x=x_{0}$ under the map $F$ is given by the graph of the function $G_{0}(\bar{x})=g\left(x_{0}, Y\left(x_{0}, \bar{x}\right)\right)$. This graph divides the plane into two parts, one above it, and one below it. If $x_{1}>x_{0}$ is given, then the image of the line $x=x_{1}$ must lie in one of these two parts. Thus we either have

$$
g\left(x_{0}, Y\left(x_{0}, \bar{x}\right)\right)>g\left(x_{1}, Y\left(x_{1}, \bar{x}\right)\right)
$$

for all $\bar{x} \in \mathbf{R}$, or we have the reverse inequality for all $\bar{x} \in \mathbf{R}$. Since $\varphi$ and $F$ are orientation preserving the latter cannot happen. Therefore $x_{0}<x_{1}$ implies that

$$
\bar{Y}\left(x_{0}, \bar{x}\right)>\bar{Y}\left(x_{1}, \bar{x}\right) \text {. }
$$


The construction of $Y$ also shows that

$$
\lim _{\bar{x} \rightarrow \pm \infty} Y(x, \bar{x})= \pm \infty
$$

and similarly $\lim _{x \rightarrow \pm \infty} Y(x, \bar{x})=\mp \infty$.

Consider a sequence of points $\left(x_{k}, y_{k}\right)(k \in \mathbf{Z})$ in the plane. They are the orbit of a point under the map $F$ if and only if they satisfy

$$
\begin{aligned}
x_{k+1} & =f\left(x_{k}, y_{k}\right) \\
y_{k} & =g\left(x_{k-1}, y_{k-1}\right)
\end{aligned}
$$

for all $k \in \mathbf{Z}$. The first equation is equivalent to $y_{k}=Y\left(x_{k}, x_{k+1}\right)$ for all $k$. If we substitute this in the second equation we get the following equivalent set of equations:

$$
\begin{aligned}
& y_{k}=Y\left(x_{k}, x_{k+1}\right) \\
& y_{k}=\bar{Y}\left(x_{k-1}, x_{k}\right) .
\end{aligned}
$$

We see that a sequence of points $\left(x_{k}, y_{k}\right)$ is an orbit of $F$ if and only if the $x$ coordinates satisfy

$$
\Delta\left(x_{k-1}, x_{k}, x_{k+1}\right)=0 \quad(\forall k \in \mathbf{Z})
$$

and the $y_{k}$ are given by $y_{k}=Y\left(x_{k}, x_{k+1}\right)$. Here we have written

$$
\Delta\left(x_{-1}, x_{0}, x_{1}\right)=Y\left(x_{0}, x_{1}\right)-\bar{Y}\left(x_{-1}, x_{0}\right) .
$$

So instead of studying orbits of the map $F$ one may as well study solutions of (2.2).

The function $\Delta$ which we have just introduced satisfies the following hypotheses. It is continuous, it is monotone increasing in both $x_{-1}$ and $x_{+1}$, and

$$
\lim _{x_{-1} \rightarrow \pm \infty} \Delta\left(x_{-1}, x_{0}, x_{1}\right)=\lim _{x_{1} \rightarrow \pm \infty} \Delta\left(x_{-1}, x_{0}, x_{1}\right)= \pm \infty \text {. }
$$

Finally, it is periodic in the sense that

$$
\Delta\left(x_{-1}, x_{0}, x_{1}\right) \equiv \Delta\left(x_{-1}+1, x_{0}+1, x_{1}+1\right)
$$

holds.

Motivated by this example we shall consider monotone recurrence relations of type $(l, m)$ for $l, m \geq 1$. We define such a relation to be one of the form

$$
\Delta\left(x_{k-1}, \ldots, x_{k+m}\right)=0 \quad(k \in \mathbf{Z})
$$

where $\Delta$ is a continuous function of $l+m+1$ variables which satisfies the following conditions:

a monotonicity $\Delta\left(x_{-l}, \ldots, x_{m}\right)$ is a nondecreasing function of all the $x_{j}$ except possibly $x_{0}$. Moreover, it is strictly increasing in the variables $x_{-1}$ and $x_{+m}$.

b periodicity $\Delta\left(x_{-1}, \ldots, x_{m}\right) \equiv \Delta\left(x_{-1}+1, \ldots, x_{m}+1\right)$.

c coerciveness $\lim _{x_{-1} \rightarrow \pm \infty} \Delta\left(x_{1}, \ldots, x_{m}\right)= \pm \infty$ and

$$
\lim _{x_{m \rightarrow \pm \infty}} \Delta\left(x_{-1}, \ldots, x_{m}\right)= \pm \infty
$$

If $\left(x_{k-1}, \ldots, x_{k+m-1}\right)$ is given then conditions a and $\underline{\mathrm{c}}$ imply that we can solve (2.4) for $x_{k+m}$. In this way we have defined a continuous map $F_{\Delta}$ from $\mathbf{R}^{1+m}$ to $\mathbf{R}^{l+m}$, given by

$$
F_{\Delta}\left(x_{k-1}, \ldots, x_{k+m-1}\right)=\left(x_{k-1+1}, \ldots, x_{k+m}\right)
$$


The conditions a and $\underline{\mathrm{c}}$ also imply that we can solve (2.4) for $x_{k-1}$ if the other variables are given. Thus the map $F_{\Delta}$ is a homeomorphism of $\mathbf{R}^{1+m}$ onto itself.

Throughout the paper we shall keep one fixed $\mathbf{Z}$ action on $\mathbf{R}^{l+m}$ in mind. It is given by

$$
\left(x_{-l}, \ldots, x_{m-1}\right)+i=\left(x_{-1}+i, \ldots, x_{m-1}+i\right)
$$

for $i \in \mathbf{Z}$. The quotient $\mathbf{R}^{1+m} / \mathbf{Z}$ is homeomorphic to $S^{1} \times \mathbf{R}^{1+m-1}$. We shall call it $\boldsymbol{A}^{1+m}$, since it generalizes the annulus $(\mathbf{R} / \mathbf{Z}) \times \mathbf{R}$ which we started with.

The periodicity condition $\underline{b}$ implies that $F_{\Delta}$ is equivalent with respect to the Z-action on $\mathbf{R}^{1+m}$, i.e. $F_{\Delta}(x+i)=F_{\Delta}(x)+i$. Hence it defines a homeomorphism on the quotient $A^{l+m}$, which we shall call $\varphi_{\Delta}$. This class of homeomorphisms is our generalization of the class of monotone twist maps of the annulus.

Our assumption that $l \geq 1$ is not a necessary assumption. If we allow $l=0$ then we can still define the maps $F_{\Delta}$ and $\varphi_{\Delta}$ as above. The only difference is that they need not be invertible anymore. Except for this all the results which we shall derive in the following sections remain true. In particular they apply to degree-one maps of the circle. If $h: \mathbf{R} \rightarrow \mathbf{R}$ satisfies $h(x+1)=h(x)+1$ then the solutions of (2.4) where we have taken

$$
\Delta\left(x_{0}, x_{1}\right)=x_{1}-h\left(x_{0}\right)
$$

are exactly the orbits of the map $h: \mathbf{R} \rightarrow \mathbf{R}$. Our map $\varphi_{\Delta}$ is the map which $h$ induces on the circle $\mathbf{R} / \mathbf{Z}$.

\section{Birkhoff orbits, and the space $X$}

Let $X$ denote the space of bi-infinite sequences of real numbers, i.e. $X=\mathbf{R}^{\mathbf{Z}}$. We equip $X$ with the product topology.

The space $X$ is also partially ordered by:

$$
x \leq y \stackrel{\text { def }}{\longleftrightarrow} x_{k} \leq y_{k} \text { for all integers } k
$$

where $x, y \in X$. The following notation is sometimes used:

$$
\begin{aligned}
& x<y \stackrel{\text { def }}{\longleftrightarrow} x \leq y \quad \text { and } x \neq y, \\
& x \ll y \stackrel{\text { def }}{\longleftrightarrow} x_{k}<y_{k} \quad \text { for all } k \in \mathbf{Z} .
\end{aligned}
$$

Given a pair of sequences $x, y \in X$ such that $x \leq y$ we define the order interval

$$
[x, y]=\{z \in X: x \leq z \leq y\} \text {. }
$$

Any order interval is homeomorphic to the product $[0,1]^{\mathrm{Z}}$, which is a compact metrizable space.

On $X$ we have a $Z \times Z$ action, $\tau$, given by

$$
\tau_{m, n}(x)_{i}=x_{i-m}+n \text {. }
$$

This action is compatible with both the topology and the partial ordering we have on $X$. 
A sequence $x \in X$ will be called a Birkhoff-sequence if for any pair of integers $(m, n)$ one either has $\tau_{m, n}(x) \geq x$ or $\tau_{m, n}(x) \leq x$. We shall denote the set of all Birkhoff sequences by $B$.

An equivalent definition of a Birkhoff sequence is the following. A sequence $x \in X$ is a Birkhoff sequence if and only if for any $i, j, k \in Z$ one has

$$
x_{i} \leq x_{j}+k \leftrightarrow x_{i+1} \leq x_{j+1}+k .
$$

Thus, if $\left\{x_{i}\right\}$ is the sequence of $x$-coordinates of an orbit of a monotone twist map, then $x$ is a Birkhoff sequence if and only if the map preserves the order on the orbit. This is the usual definition of a 'Birkhoff-orbit' (see [Ka1]).

It is known that if $x \in B$ is a Birkhoff sequence, then $x$ has a rotation number, i.e. the limit

$$
\lim _{k \rightarrow \pm \infty} \frac{x_{k}}{k}=\omega \stackrel{\text { def }}{=} \omega(x)
$$

exists. In fact one has the following inequalities:

$$
x_{0}+\lfloor\omega k\rfloor \leq x_{k}<x_{0}+\lfloor\omega k\rfloor+1 \quad(k \in Z)
$$

where $\lfloor\omega k\rfloor$ is the largest integer below $\omega k$.

The set of Birkhoff sequences, $B$, which is the intersection of the sets $\left\{x \in X: \tau_{m, n}(x) \geq x\right.$ or $\left.\tau_{m, n}(x) \leq x\right\}$, is a closed subset of $X$.

The whole set $B$ is not compact, but the inequalities (3.1) imply that for any two constants $\alpha, \beta>0$ the closed set

$$
\left\{x \in B|| x_{0} \mid \leq \alpha \text { and }|\omega(x)| \leq \beta\right\}
$$

is contained in an order interval, and therefore compact.

We shall occasionally say something about periodic orbits 'of type $(p, q)$ '.

If the map $\varphi_{\Delta}$ of $\mathbf{R}^{l+m} / \mathbf{Z}$ has a periodic point $P$ with period $q$, then some lift $\left(x_{-l}, \ldots, x_{m-1}\right) \in \mathbf{R}^{1+m}$ of $P$ will almost be periodic in the sense that the corresponding sequence $\left\{x_{k}, k \in Z\right\} \in X$ satisfies

$$
x_{k+q}=x_{k}+p \quad(k \in \mathbf{Z})
$$

for some $p \in \mathbf{Z}$. Another way of saying this is $x=\tau_{p, q}(x)$.

By definition any sequence $x \in X$ will be said to be periodic of type $(p, q)$ if it satisfies (3.2).

If $x \in X$ is periodic of type $(p, q)$ and if $x$ is also a Birkhoff orbit or sequence then $x$ is periodic of type $\left(p_{0}, q_{0}\right)$ where $p=l \cdot p_{0}, q=l \cdot q_{0}$ and $l=\operatorname{gcd}(p, q)$.

Indeed, $x \in B$ implies that we have $\tau_{p_{0}, q_{0}}(x) \geq x$ or $\tau_{p_{0}, q_{0}}(x) \leq x$. Suppose the first holds, and for some $i \in \mathbf{Z}$ we have

$$
\tau_{p_{0}, q_{0}}(x)_{i}>x_{i} \text {, i.e. } x_{i-q_{0}}+p_{0}>x_{i} .
$$

Then $\tau_{p_{0}, q_{0}}(x) \geq x$ implies that for all $k \in \mathbf{Z}$

$$
x_{i-k q_{0}}+k p_{0} \geq x_{i-(k-1) q_{0}}+(k-1) p_{0}
$$

so that $x_{i}=x_{i-1 q_{0}}+l p_{0}>x_{i}$, a clear contradiction. If we did not have $\tau_{p_{0}, q_{0}}(x) \geq x$ but $\tau_{p_{0}, q_{0}}(x) \leq x$ instead then the same arqument would show that we still have $\tau_{p_{0}, q_{0}}(x)=$ $x$. 


\section{Subsolutions and supersolutions}

Let $\Delta \in C\left(\mathbf{R}^{l+m+1}\right)$ be a monotone recurrence relation of type $(l, m)$ as we defined them in $\$ 2$.

A sequence $x \in X$ is called a subsolution for $\Delta$ iff

$$
\Delta\left(x_{j-l}, \ldots, x_{j+m}\right) \geq 0 \quad(\forall j \in \mathbf{Z})
$$

holds. Similarly, a supersolution is a sequence $x \in X$ such that

$$
\Delta\left(x_{j-1}, \ldots, x_{j+m}\right) \leq 0 \quad(\forall j \in \mathbf{Z})
$$

holds.

The next lemma states some of the elementary properties of sub- and supersolutions.

LEMMA 4.1. (a) The set of subsolutions for $\Delta$ is closed in $X$. The same holds for supersolutions.

(b) If $\left\{x^{(\alpha)}\right\}_{\alpha \in \mathscr{A}}$ is a family of subsolutions which is bounded from above (w.r.t. the partial ordering on $X)$ then $\sup x^{(\alpha)}$ defined by

$$
\left(\sup _{\alpha} x^{(\alpha)}\right)_{i}=\sup _{\alpha}\left(x_{i}^{(\alpha)}\right)
$$

is a subsolution.

Analogously, a family of supersolutions $x^{(\alpha)}$ which is bounded from below has an infimum, and inf $x^{(\alpha)}$ is again a supersolution.

Proof. Part (a) follows immediately from the continuity of the function $\Delta$.

To prove part (b), let $x^{(\alpha)}$ be a family of subsolutions, indexed by $\alpha \in \mathscr{A}$, and let $\bar{x}=\sup x^{(\alpha)}$. Then for each $j \in \mathbf{Z}$ there exists a sequence of $\alpha_{j k}$ 's for which

$$
x_{j}^{\left(\alpha_{j k}\right)} \geq \bar{x}_{j}-2^{-k} \quad(k=1,2,3, \ldots, j \in Z)
$$

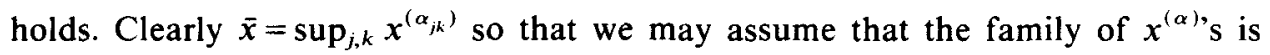
countable. Moreover, if one defines

$$
x^{(N)}=\sup \left(x^{\left(\alpha_{j k}\right)}|0 \leq k,| j \mid \leq N\right)
$$

then, as $N \uparrow \infty$, the $x^{(N)}$ converge in the product topology on $X$ to $\bar{x}$. In view of part (a) of this lemma we only have to consider the case in which the number of $x^{(\alpha)}$ 's is finite. In turn, this may be reduced to the case of only two $x^{(\alpha)}$, s, by means of an induction argument.

So consider two subsolutions $x, y \in X$ and let $\bar{x}=\sup (x, y)$ be their maximum or supremum. Let $j \in \mathbf{Z}$ be given, and assume that $x_{j} \geq y_{j}$. Then for any nonzero integer $k$ in the range $-l \leq k \leq+m$ one has $\bar{x}_{j+k} \geq x_{j+k}$, so that the monotonicity of $\Delta$ (i.e. hypothesis $2.5 a$ ) implies that

$$
\Delta\left(\bar{x}_{j-l}, \ldots, \bar{x}_{j+m}\right) \geq \Delta\left(x_{j-l}, \ldots, x_{j+m}\right) \geq 0 .
$$

If $x_{j} \leq y_{j}$ then one has, for the same reasons,

$$
\Delta\left(\bar{x}_{j-l}, \ldots, \bar{x}_{j+m}\right) \geq \Delta\left(y_{j-l}, \ldots, y_{j+m}\right) \geq 0 .
$$

In either case we obtain $\Delta\left(\bar{x}_{j-l}, \ldots, \bar{x}_{j+m}\right) \geq 0$ for arbitrary $j$, so that $\bar{x}$ is a subsolution. This finishes the proof of the lemma.

The next result shows how subsolutions and supersolutions may be used to construct actual solutions of the recurrence relation (2.4). 
THEOREM 4.2. Let $\underline{x}, \bar{x} \in X$ be a sub- and a supersolution, respectively, which are ordered: $\underline{x} \leq \bar{x}$. Then there is at least one solution of (2.4), say $x$, between $\underline{x}$ and $\bar{x}$, i.e. for which $x \leq x \leq \bar{x}$ holds.

Proof. Define

$$
\underline{S}=\{x \in X \mid x \text { is a subsolution and } x \leq \bar{x}\} .
$$

Clearly $\underline{S}$ is bounded from above, so that

$$
x=\sup \underline{S}
$$

exists. Since $\underline{x} \in \underline{S}$ we have $\underline{x} \leq x<\bar{x}$. By our previous lemma $x$ is again a subsolution, and therefore $x \in \underline{S}$. We have for every integer $j$

$$
\Delta\left(x_{j-l}, \ldots, x_{j+m}\right) \geq 0 \text {. }
$$

We claim that equality holds for all $j$, i.e. that $x$ is a solution of (2.4).

To reach a contradiction assume that for some $j$ strict inequality holds in (4.2). Then we must have $x_{j}<\bar{x}_{j}$, or $x_{j}=\bar{x}_{j}$.

In the first case we define

$$
x_{k}^{\varepsilon}= \begin{cases}x_{j}+\varepsilon & \text { if } j=k \\ x_{j} & \text { if } j \neq k\end{cases}
$$

The monotonicity of $\Delta$ implies that $\Delta\left(x_{k-1}^{\varepsilon}, \ldots, x_{k+m}^{\varepsilon}\right) \geq 0$ whenever $k \neq j$ and $\varepsilon \geq 0$. Since we have strict inequality in (4.2) and $\Delta$ is continuous we also have $\Delta\left(x_{j-l}^{\varepsilon}, \ldots, x_{j+m}^{\varepsilon}\right) \geq 0$ if $\varepsilon>0$ is small enough. So for small $\varepsilon>0, x^{\varepsilon}$ beongs to $\underline{S}$, but $x^{\varepsilon}>x$ which is a contradiction.

In the other case, i.e. $x_{j}=\bar{x}_{j}$, we observe that, due to the monotonicity of $\Delta$,

$$
\Delta\left(\bar{x}_{j-1}, \ldots, \bar{x}_{j+m}\right) \geq \Delta\left(x_{j-1}, \ldots, x_{j+m}\right)>0 .
$$

But $\bar{x}$ is a supersolution so that we have a contradiction again.

We must conclude therefore that $x=\sup \underline{S}$ is a solution of the recurrence relation.

It should be clear from the proof that if we had defined

$$
\bar{S}=\{x \in X \mid x \geq x \text { and } x \text { is a supersolution }\}
$$

then inf $\bar{S}$ would also be a solution of the recurrence relation. In general one expects inf $\bar{S}$ and $\sup \underline{S}$ to be different.

The theorem can also be used to construct Birkhoff orbits or sequences.

ADDENDUM 4.3. If at least one of $\underline{x}$ and $\bar{x}$ is a Birkhoff sequence, then there exists a solution $x$ of (2.4) which lies between $\underline{x}$ and $\bar{x}$, and is a Birkhoff sequence.

Proof. Let $\bar{x}$ be a Birkhoff sequence. Then we shall prove that $x=\sup (\underline{S})$ also is a Birkhoff sequence.

Let $m, n$ be given integers. Then we have $\tau_{m, n}(\bar{x}) \leq \bar{x}$ or $\tau_{m, n}(\bar{x}) \geq \bar{x}$.

In the first case we have, for any $y \in \underline{S}$,

$$
\tau_{m, n}(y) \leq \tau_{m, n}(\bar{x}) \leq \bar{x}
$$

so that $\tau_{m, n}(y) \in \underline{S}$ (note that the translations $\tau_{m, n}$ preserve the ordering on $X$, and that $y$ is a sub(super)solution if and only if $\tau_{m, n}(y)$ is one). 
Thus $\tau_{m, n}(\underline{S}) \subset \underline{S}$ and

$$
\tau_{m, n}(x)=\tau_{m, n}(\sup \underline{S})=\sup \tau_{m, n}(\underline{S}) \leq \sup \underline{S}=x .
$$

In the other case we have $\tau_{-m,-n}(\bar{x}) \leq \bar{x}$, so that the same argument shows that $\tau_{-m,-n}(x) \leq x$ or, $\tau_{m, n}(x) \geq x$.

The conclusion is, that for any $m, n \in \mathbf{Z}$, one has either $\tau_{m, n}(x) \leq x$ or $\tau_{m, n}(x) \geq x$, so that $x$ is a Birkhoff sequence.

If $\underline{x}$ is not a Birkhoff sequence, but $\bar{x}$ is then one shows in the same way that $\inf (\bar{S})$ is a Birkhoff sequence.

ADDENDUM 4.4. If at least one of $\bar{x}$ or $\underline{x}$ is periodic of type $(p, q)$, then there exists a solution $x$ of (2.4) which lies between $x$ and $\bar{x}$, and is periodic of type $(p, q)$.

Proof. Let $\bar{x}$ be periodic of type $(p, q)$. Then $\tau_{p, q}(\bar{x})=\bar{x}$, and therefore $y \in \underline{S}$ implies $\tau_{p, q}(y) \in \underline{S}$. But then $\tau_{p, q}(\sup \underline{S})=\sup \underline{S}$, so that $\sup \underline{S}$ is periodic of type $p, q$.

Likewise, if $\underline{x}$ is periodic of type $(p, q)$ then so is inf $\bar{S}$.

\section{A generalization of Hall's theorem}

In [Ha1] Hall proved that any monotone twist map, which has a periodic orbit of type $(p, q)$, must have a Birkhoff orbit of the same type. The following theorem generalizes this result.

THEOREM 5.1. Let $x \in X$ be a solution of the monotone recurrence relation (2.4) for which one can find a real number $\omega$ such that

$$
M=\sup _{k \in \mathbf{Z}}\left|x_{k}-k \cdot \omega\right|<\infty .
$$

Then (2.4) has a Birkhoff solution whose rotation number equals $\omega$.

Proof. Any translate $\tau_{m, n}(x)$ of $x$ is also a solution. Our assumption implies that

and

$$
\underline{x}=\sup \left\{\tau_{m, n}(x) \mid n \leq m \cdot \omega\right\}
$$

$$
\bar{x}=\inf \left\{\tau_{m, n}(x) \mid n \geq m \cdot \omega\right\}
$$

both exist and that

$$
\begin{gathered}
0 \leq \underline{x}_{k}-x_{k}=\sup _{n \leq \omega m}\left(x_{k-m}+n-x_{k}\right) \leq 2 M \\
0 \leq x_{k}-\bar{x}_{k} \leq 2 M
\end{gathered}
$$

holds. So if we define $\underline{y}_{k}=\underline{x}_{k}-2 M$ and $\bar{y}_{k}=\bar{x}^{k}+2 M$ then $\underline{y}$ is a subsolution, $\bar{y}$ is a supersolution and $y \leq \bar{y}$.

Moreover, $\underline{y}$ and $\bar{y}$ are Birkhoff sequences.

To see this let $r, s \in \mathbf{Z}$ be given and consider $\tau_{r, s}(\underline{x})$ :

$$
\begin{aligned}
\tau_{r, s}(x) & =\sup \left(\tau_{m+r, n+s}(x) \mid n \leq m \omega\right) \\
& =\sup \left(\tau_{m, n}(x) \mid n \leq m \omega+s-r \omega\right) \\
& \geq \underline{x} \text { if } s \geq r \omega \\
& \leq \underline{x} \text { if } s \leq r \omega .
\end{aligned}
$$


Thus $\underline{x}$ is a Birkhoff sequence, and therefore $\underline{y}$ is one too. A similar argument proves that $\bar{y}$ is a Birkhoff sequence.

The addendum to Theorem 4.2 implies that the recurrence equation (2.4) has a Birkhoff solution $y$, between $\underline{y}$ and $\bar{y}$. Clearly $y$ must have rotation number $\omega$.

\section{Quasiperiodic orbits with prescribed rotation number}

In this section we assume that for some $\omega_{0}<\omega_{1}$ there exist a subsolution $\underline{x}$ and a supersolution $\bar{x}$ of (2.4) such that

$$
\begin{aligned}
& \liminf _{k \rightarrow \infty} \frac{\bar{x}_{k}}{k} \geq \omega_{1}, \\
& \limsup _{k \rightarrow-\infty} \frac{\underline{x}_{k}}{k} \geq \omega_{1}, \\
& \liminf _{k \rightarrow-\infty} \frac{\bar{x}_{k}}{k} \leq \omega_{0}, \\
& \limsup _{k \rightarrow+\infty} \frac{\underline{x}_{k}}{k} \leq \omega_{0},
\end{aligned}
$$

holds. The main result of this section is:

THEOREM 6.1. For any $\omega \in\left[\omega_{0}, \omega_{1}\right]$ there is a Birkhoff solution of (2.4) with rotation number $\omega$.

Proof. The inequalities (6.1) imply that

$$
\liminf _{k \rightarrow \pm \infty}|k|^{-1} \cdot\left(\bar{x}_{k}-\omega \cdot k\right) \geq \min \left(\omega_{1}-\omega, \omega-\omega_{0}\right)>0
$$

and

$$
\limsup _{k \rightarrow \pm \infty}|k|^{-1} \cdot\left(\underline{x}_{k}-\omega \cdot k\right) \leq \max \left(\omega-\omega_{1}, \omega_{0}-\omega\right)<0 .
$$

Hence there is an integer $N \geq 0$ such that for all $k \in \mathbf{Z}$ one has:

$$
\bar{x}_{k} \geq \omega k-N \text { and } \underline{x}_{k} \leq \omega k+N \text {. }
$$

Now define

$$
\begin{aligned}
& \bar{y}=\inf \left(\tau_{m, n}(\bar{x})+N \mid n \geq m \omega\right) \\
& \underline{y}=\sup \left(\tau_{m, n}(\underline{x})-N \mid n \leq m \omega\right) .
\end{aligned}
$$

Then, on the ground of arguments similar to those which were used in the proof of Theorem 5.1, one concludes that $\bar{y}$ is a Birkhoff supersolution with rotation number $\omega$, and that $\underline{y}$ is a Birkhoff subsolution with the same rotation number.

Furthermore (6.2) implies that

$$
\bar{y}_{k} \geq \omega k \geq \underline{y}_{k},
$$

i.e. that $y \leq \bar{y}$. By Theorem 4.2 and the Addendum 4.3 there must be a Birkhoff solution $y$ between $\underline{y}$ and $\bar{y}$. This Birkhoff solution has rotation number $\omega$. 


\section{A criterion for positive entropy}

Under the hypotheses of $\S 6$ we can construct a great number of orbits of $\varphi_{\Delta}$. This construction leads us to the following conclusion.

THEOREM 7.1. Let the monotone recurrence relation (2.4) have a sub-and a supersolution, $\underline{x}$ and $\bar{x}$ respectively, which satisfy (6.1) for some $\omega_{0}<\omega_{1}$.

Then the map $\varphi_{\Delta}$ has positive topological entropy. In fact there is a compact subset $K$ of $A^{1+m}$ such that some iterate of $\varphi_{\Delta}$ leaves $K$ invariant, and has a Bernoulii shift as a factor, when restricted to $K$.

Since the existence of $K$ implies positivity of the topological entropy of $\varphi_{\Delta}$ we only have to construct it to prove the theorem.

The construction proceeds in three steps. In the first step we use $\underline{x}$ and $\bar{x}$ to construct a nicer pair of sub- and supersolutions $\underline{w}$ and $\bar{w}$, which are wedge shaped, as in figure 1 .

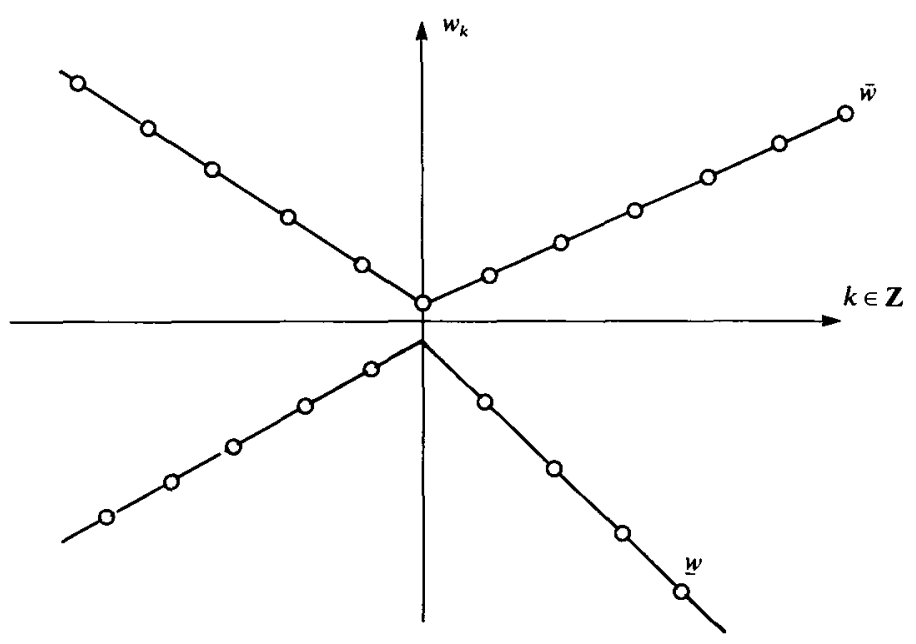

FIGURE 1. Graphs of $\underline{w}$ and $\bar{w}$.

Then, in the next step we consider a biinfinite sequence of translates of $\underline{w}$ and $\bar{w}$. These translates of our original wedges are chosen in such a manner that, if $\underline{W}$ denotes the supremum of all subsolutions in question, and $\bar{W}$ the infimum of the translated supersolutions, then $\underline{W} \leq \bar{W}$ and from the results in section four we know that there is a solution $W$ between $\underline{W}$ and $\bar{W}$. It turns out that we have so much freedom in choosing the translated sub- and supersolutions that we can let the constructed solution $W$ follow any 'zig-zag pattern' we like (see figure 2 ).

Now let us fill in the details.

The wedge like sub-and supersolution. Choose two rational numbers $\rho_{0}, \rho_{1}$ such that

$$
\omega_{0}<\rho_{0}<\rho_{1}<\omega
$$




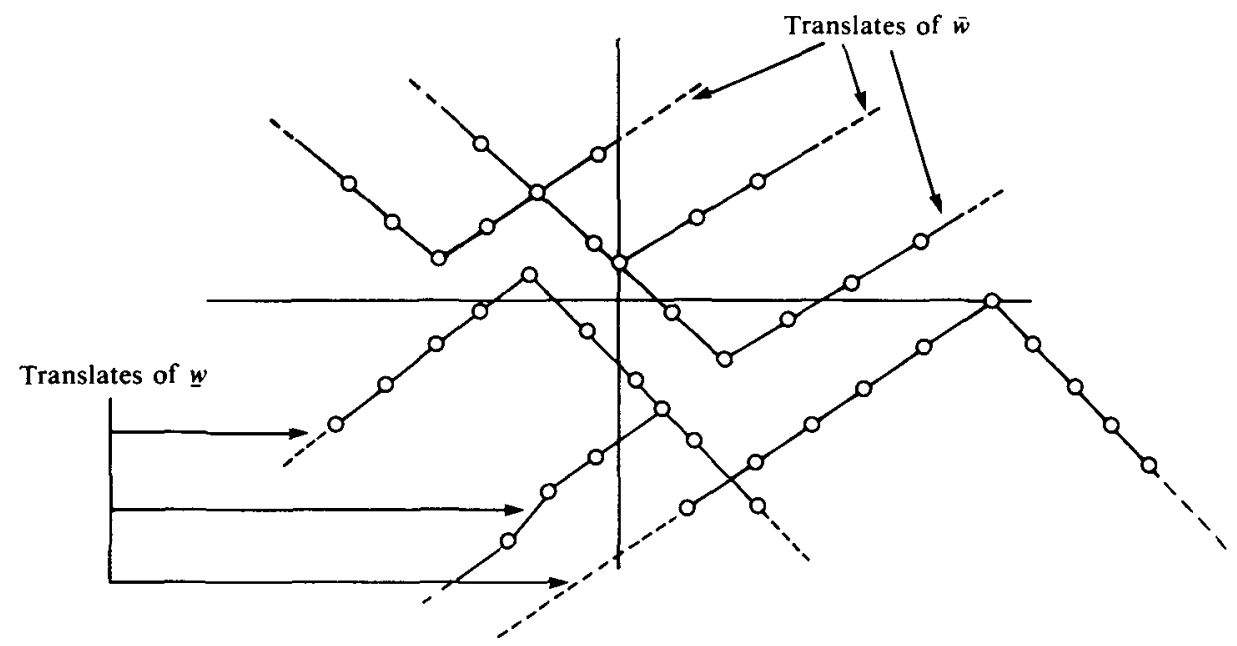

Figure 2.

holds, and define the functions

$$
\begin{aligned}
& z(t)=\rho_{0} t_{+}-\rho_{1} t_{-}, \\
& Z(t)=\rho_{1} t_{+}-\rho_{0} t_{-},
\end{aligned}
$$

where $t_{+}=\max (t, 0)$ and $t_{-}=\max (-t, 0)$.

From our sub- and supersolution $\underline{x}$ and $\bar{x}$ we construct new sub- and supersolutions

$$
\begin{aligned}
& \underline{w}=\sup \left\{\tau_{m, n}(\underline{x}): n \leq z(m)\right\} \\
& \bar{w}=\inf \left\{\tau_{m, n}(\bar{x}): n \geq Z(m)\right\} .
\end{aligned}
$$

Lemma 4.1 assures us that $\underline{w}$ is a subsolution, and that $\bar{w}$ is a supersolution.

Our hypothesis (6.1) on $x$ and $\bar{x}$ implies that

and

$$
\bar{x}_{k} \geq Z(k)-M
$$

$$
\underline{x}_{k} \leq z(k)+M
$$

holds for all $k \in \mathbf{Z}$, with $M$ independent of $k$. For any $(m, n)$ with $n \leq z(m)$ one has

$$
\begin{aligned}
\left(\tau_{m, n} \underline{x}\right)_{k} & =\underline{x}_{k-m}+n \\
& \leq z(k-m)+M+z(m) \\
& \leq z(k)+M
\end{aligned}
$$

(note that $-z(t)$ is sub additive, so that $-z(k) \geq-z(k-m)-z(m)$ ). Thus after taking the supremum over all $m, n$ with $n \leq z(m)$ one finds

$$
\underline{w}_{k} \leq z(k)+M \text {. }
$$

Furthermore, if one puts $m=k$ and $n=\lfloor z(k)\rfloor$ (the largest integer below $z(k)$ ) then

$$
\underline{w}_{k} \geq\left(\tau_{m, n} \underline{x}\right)_{k}=\underline{x}_{0}+\lfloor z(k)\rfloor \text {. }
$$


Without loss of generality we may assume that the constant $M$ is so large that $M>\left|\underline{x}_{0}\right|+1$. Then we have just proved that

$$
\left|\underline{w}_{k}-z(k)\right| \leq M
$$

for all $k \in \mathbf{Z}$.

A similar argument gives

$$
\left|\bar{w}_{k}-Z(k)\right| \leq M
$$

(it may be necessary to increase the constant $M$ one more time).

Construction of a zig-zag solution. In the previous step we had chosen two rational numbers $\rho_{0}, \rho_{1}$. Let $Q$ be a common multiple of their denominators, so that $\rho_{0} Q$ and $\rho_{1} Q$ are integers. We choose $Q$ so large that

$$
\left(\rho_{1}-\rho_{0}\right) \cdot Q \geq 4 M \text {. }
$$

Let $\left\{e_{k} ; k \in \mathbf{Z}\right\}=e$ be an arbitrary biinfinite sequence of zeroes and ones (i.e. $e_{k}=0$ or $e_{k}=1$ for all $\left.k \in Z\right)$. Given such a sequence we define two functions, $\chi_{e}(t)$ and $\zeta_{e}(t)$. On the interval $j Q \leq t<(j+1) Q$ we define

$$
\chi_{e}(t)=\rho_{e_{j}} \text {, }
$$

i.e. $\rho_{1}$ if $e_{j}=1$ and $\rho_{0}$ if $e_{j}=0$. The other function is given by

$$
\zeta_{e}(t)=\int_{0}^{t} \chi_{e}(s) d s .
$$

For any integer $j, \zeta_{e}(j Q)$ is an integer, and we always have the inequalities

$$
z(t) \leq \zeta_{e}(t) \leq Z(t)
$$

whatever $e$ is.

Now define

$$
\begin{aligned}
& \underline{W}_{e}=\sup \left\{\tau_{j Q, \zeta_{e}(j Q)}(\underline{w}) \mid j \in \mathbf{Z}\right\} \\
& \bar{W}_{e}=\inf \left\{\tau_{j Q, \zeta_{e}(j Q)}(\bar{w}) \mid j \in \mathbf{Z}\right\} .
\end{aligned}
$$

Proposition 7.2.

$$
\begin{aligned}
& \left|\underline{W}_{e, k}-\zeta_{e}(k)\right| \leq M \\
& \left|\bar{W}_{e, k}-\zeta_{e}(k)\right| \leq M .
\end{aligned}
$$

Proof. For any $m \in \mathbf{X}$ we have

$$
\begin{aligned}
{\left[\tau_{j Q, \zeta_{e}(j Q)}(\underline{w})\right]_{m}-\zeta_{e}(m) } & =\underline{w}_{m-j Q}+\zeta_{e}(j Q)-\zeta_{e}(m) \\
& =\underline{w}_{m-j Q}-z(m-j Q)+z(m-j Q)-\int_{j Q}^{m} \chi_{e}(s) d s \\
& \leq \underline{w}_{m-j Q}-z(m-j Q) \\
& \leq M,
\end{aligned}
$$

where we have used (7.2) in the last step, and the inequality

$$
z(t) \leq \int_{x}^{x+t} \chi_{e}(s) d s \quad(\forall t \in \mathbf{R}, x \in \mathbf{R})
$$


(which follows from $\rho_{0} \leq \chi_{e} \leq \rho_{1}$ ) in the second last step. This proves one half of the first inequality. To prove the second half we choose an integer $j$ such that $j Q \leq m<(j+1) Q$.

If $e_{j}=0$ then

$$
\begin{aligned}
\underline{W}_{e, m} & \geq \tau_{j Q, \zeta_{e}(j Q)}(\underline{w})_{m} \\
& =\underline{w}_{m-j Q}+\zeta_{e}(j Q) \\
& \geq z(m-j Q)+\zeta_{e}(j Q)-M \\
& =\rho_{0}(m-j Q)+\zeta_{e}(j Q)-M \\
& =\zeta_{e}(j Q)-M .
\end{aligned}
$$

In case $e_{j}=1$ one compares $\underline{W}_{e, m}$ with $\tau_{(j+1) Q, \zeta_{e}((j+1) Q)}(\underline{w})_{m}$ to arrive at the same conclusion.

Therefore the first inequality in the Proposition holds. The proof of the second inequality is analogous to that of the first.

It follows immediately from this proposition that $\underline{W}_{e}-M$ and $\bar{W}_{e}+M$ are a suband supersolution, and the $\underline{W}_{e}-M \leq \bar{W}_{e}+M$, so that there must be at least one solution $W$ of (2.4) which lies between $\underline{W}_{e}-M$ and $\bar{W}_{e}+M$.

Construction of the set $K$. Let $\Sigma_{e}$ denote the set of solutions of (2.4) such that $\underline{W}_{e}-M \leq W \leq \bar{W}_{e}+M$. We have just seen that $\Sigma_{e}$ is nonempty.

Let $\Sigma$ be the union of all $\Sigma_{e}$ where $e$ ranges over all possible $\{0,1\}$-sequences.

To define $K$, we recall that it has to be a subset of $\mathbf{R}^{l+m} / \mathbf{Z}$, where $\mathbf{Z}$ acts on $\mathbf{R}^{l+m}$ via $\left(x_{-l}, \ldots, x_{m-1}\right)+1=\left(x_{-1}+1, \ldots, x_{m-1}+1\right)$.

Then we put

and

$$
K_{e}=\left\{\left(x_{-l}, \ldots, x_{m-1}\right) \bmod \mathbf{Z} \mid x \in \Sigma_{e}\right\}
$$

$$
K=\left\{\left(x_{-1}, \ldots, x_{m-1}\right) \bmod \mathbf{Z} \mid x \in \Sigma\right\} .
$$

Proposition 7.3. $K$ and the $K_{e}$ are compact.

Proof. Since $\Sigma_{e}$ is contained in the order interval $\left[\underline{W}_{e}-M, \bar{W}^{e}+M\right]$ it is precompact in the product topology on $X$. Since it is closed $\Sigma_{e}$ is in fact compact.

Each $K_{e}$ is the image of $\Sigma_{e}$ under the projection map from $X$ to $\mathbf{R}^{1+m} / \mathbf{Z}$, which is defined by

$$
\pi(x)=\left(x_{-1}, \ldots, x_{m-1}\right)(\bmod Z)
$$

for $x \in X$. Thus $K_{e}=\pi\left(\Sigma_{e}\right)$ is also compact (the projection map is continuous).

We claim that $\Sigma$ is also compact. To verify this we observe that $\Sigma$ is contained in the order interval $\left\{x \in X \mid z(k)-M \leq x_{k} \leq Z(k)+M\right\}$ and therefore is precompact. It remains to show that $\Sigma$ is closed. Since $X$ is metrizable we only have to check for sequential closedness. So let $\left\{w^{(m)}\right\}_{m \geq 1}$ be a sequence in $\Sigma$ which converges to $w^{*} \in X$. Then for every $m \geq 1$ there is a $\{0,1\}$ sequence $e^{(m)}=\left\{e_{k}^{(m)}\right\}_{k \in \mathbf{Z}}$ such that $w^{(m)} \in \Sigma_{e}(m)$. Now, by passing to a subsequence if necessary, we can arrange things so that for any $k$ the sequence $\left\{e_{k}^{(m)}\right\}_{k \geq 1}$ is eventually constant. If one runs through the definition of the $\underline{W}_{e}$ and $\bar{W}_{e}$ one more time then one sees that this implies that 
the $\underline{W}_{e}(m)$ and $\bar{W}_{e}(m)$ converge to $\underline{W}_{e^{*}}$ and $\bar{W}_{e^{*}}$ respectively (where

$$
\left.e_{k}^{*}=\lim _{m \rightarrow \infty} e_{k}^{(m)}\right) \text {. }
$$

It follows that $w^{*} \in \Sigma_{e^{*}}$ and thus $w^{*} \in \Sigma$.

Hence $\Sigma$ is closed, and even compact.

We may conclude the proof of the proposition by noting that $K=\pi(\Sigma)$ is the continuous image of a compact set, and therefore also compact.

Proposition 7.4. The sets $K_{e}$ are pair wise disjoint.

Proof. Let $\left(x_{-l}, \ldots, x_{m-1}\right) \in \mathbf{R}^{1+m}$ represent a point in $K_{e}$. Then we can form its orbit under the diffeomorphism $F_{\Delta}$ (defined in $\S 2$-it is a lift of $\varphi_{\Delta}$ ), which gives us a biinfinite sequence $\left\{x_{j}\right\}_{j \in Z}=x$.

On the other hand, there must be an $\tilde{x} \in \Sigma$ such that $\left(x_{-l}, \ldots, x_{m-1}\right)(\bmod Z)=\pi(\tilde{x})$. Since $\tilde{x}$ is a solution of (2.4) it is uniquely determined by its components $\left(\tilde{x}_{-l}, \ldots, \tilde{x}_{m-1}\right)$, and we see that $\tilde{x}=x(\bmod Z)$.

By Proposition 7.2 we see that

$$
\begin{aligned}
\left|\left(\tilde{x}_{(j+1) Q}-\tilde{x}_{j \cdot Q}\right)-\rho_{e_{j}} \cdot Q\right| & \leq 2 M+\left|\zeta_{e}((j+1) Q)-\zeta_{e}(j Q)-\rho_{e_{j}} \cdot Q\right| \\
& =2 M .
\end{aligned}
$$

We had assumed that $\left(\rho_{1}-\rho_{0}\right) \cdot Q>4 M$, so that the inequality cannot be true for both $e_{j}=1$ and $e_{j}=0$. In other words, given our point in $K_{e}$ we can find $\tilde{x}$, and from $\tilde{\boldsymbol{x}}$ we can compute the sequence $e$. Hence two different $K_{e}$ 's cannot overlap.

The proof of the last proposition also shows that we have a continuous mapping $\varepsilon: K \rightarrow C$ where $C=\{0,1\}^{\mathrm{z}}$ is the space of all $\{0,1\}$ sequences equipped with the product topology ( $C$ is homeomorphic to the Cantor set).

The mapping $\varepsilon$ is surjective.

On $C$ we have a homeomorphism, called the shift. It acts by $\sigma(e)_{k}=e_{k+1}$. Using the fact that if $w \in \Sigma_{e}$, then

$$
\tilde{w}=\tau_{-Q,-P}(w) \quad \text { with } P=-\rho_{e_{j}} \cdot Q
$$

belongs to $\Sigma_{\sigma(e)}$, and also the fact that

$$
\left(\varphi_{\Delta}\right)^{Q}(\pi(w))=\pi(\tilde{w})
$$

one can verify that

(i) $K$ is invariant under $\left(\varphi_{\Delta}\right)^{Q}$

(ii) $\varepsilon \cdot\left(\varphi_{\Delta}\right)^{Q}=\sigma \cdot \varepsilon$, i.e. $\left(\varphi_{\Delta}\right)^{Q}$ maps $K_{e}$ onto $K_{\sigma(e)}$.

We can restate this by saying that $(C, \sigma)$ is a factor of $\left(K,\left(\varphi_{\Delta}\right)^{Q}\right)$. By a standard result of ergodic theory this implies that $\varphi_{\Delta}$ has positive topological entropy (see [Wa]).

We conclude this section with the following observation. If $e \in C$ is a periodic sequence then the corresponding sub- and supersolution, $\underline{W}_{e}$ and $\bar{W}_{e}$, are also periodic of type $(k, l)$ for suitable $k$ and $l$. By the second addendum to Theorem 4.2 we know that there must be a $(k, l)$-periodic solution $W_{e}$ between $W_{e}$ and $\vec{W}_{e}$. Thus we obtain the existence of many periodic orbits of the map $\varphi_{\Delta}$.

Indeed, if the sequence $e \in C$ has period $k_{0}$, then $k=k_{0} \cdot Q$. It follows that the number of periodic orbits of $\varphi_{\Delta}$ whose period divides $k_{0} \cdot Q$ is at least $2^{k_{0}}$. 


\section{Twist maps with zero entropy}

Using the criterion for positivity of the topological entropy which we derived in the last section, we now study monotone twist maps of the (two dimensional) annulus $A=\mathbf{R} \times S^{1}$, whose entropy vanishes.

Let $\varphi$ be such a map, and let $F$ be its lift to $\mathbf{R}^{2}$. We assume it satisfies the conditions which were described in $\S 2$. To any point $P \in A$ we associate the sequence $\left\{x_{k}(P)\right\}$ where $x_{k}(P)$ is the $x$ coordinate of $F^{k}\left(P^{\prime}\right)$ and $P^{\prime}$ is a lift of $P$. The sequence $x_{k}(P)$ depends on the particular lift $P^{\prime}$ of $P$ which we choose, but the sequence $x_{k}(P)-x_{0}(P)$ does not.

We define the forward- and backward-rotation numbers of $P$ to be the limits

$$
\rho_{ \pm}(P)=\lim _{k \rightarrow \infty} \frac{x_{k}(P)-x_{0}(P)}{k}
$$

if they exist. We shall allow $\rho_{ \pm}(P)$ to be $+\infty$ or $-\infty$.

The main result of this section is:

THEOREM 8.1. If the map $\varphi$ has zero topological entropy, then every $P \in A$ has a forward and backward rotation number.

Our other result is:

THeOREM 8.2. If the map $\varphi$ has zero topological entropy and $P$ is a periodic point of type $(p, q)$ with $\operatorname{gcd}(p, q)=1$, then the orbit of $P$ is a Birkhoff orbit.

This was originally proven by Boyland, using Thurston's classification of surface diffeomorphisms.

We begin our proof of these theorems by assuming that we have transformed the problem of finding orbits of the $\operatorname{map} \varphi$ to the problem of finding solutions to the recurrence equation (2.2).

Let $x, y \in X$ be two biinfinite sequences. We shall say that $x$ and $y$ intersect at the integer $k$ if either

$$
\left(y_{k}-x_{k}\right)\left(y_{k+1}-x_{k+1}\right)<0
$$

or

$$
y_{k}=x_{k} \quad \text { and }\left(y_{k-1}-x_{k-1}\right)\left(y_{k+1}-x_{k+1}\right)<0
$$

holds. $\dagger$

Clearly $x$ and $y$ are ordered if and only if they do not intersect at any integer $k$. In particular $x$ is a Birkhoff sequence iff it does not intersect any of its translates, $\tau_{m, n}(x)$.

Given a sequence $x \in X$, it can happen that, for some pair of integers $(m, n)$, with $m>0, x$ and $\tau_{m, n}(x)$ intersect at an infinite number of positive integers $k_{1}, k_{2}, k_{3}, \ldots$

The idea of looking at the intersections of the graphs of two sequences $x^{1}, x^{2} \in X$ is not new, as the referee kindly pointed out to me. Indeed, V. Bangert uses this notion in his paper [Ba], and it was also used in [A]. 
We shall denote the set of $(m, n)$ for which this occurs by $J(x)$. In addition we define the corresponding set of ratios:

$$
R(x)=\left\{\frac{n}{m} \mid(m, n) \in J(x)\right\} \subset Q .
$$

LEMMA 8.3. If $R(x)$ is finite, then $x$ has a forward rotation number.

Proof. Define

$$
\bar{\rho}=\limsup _{k \rightarrow \infty} \frac{x_{k}}{k} \quad \text { and } \quad \underline{\rho}=\liminf _{k \rightarrow \infty} \frac{x_{k}}{k} .
$$

If $x$ does not have a forward rotation number, then $\underline{\rho}<\bar{\rho}$, and there has to be a rational number $a / b$ such that $\rho<a / b<\bar{\rho}$. Moreover we can choose $a / b$ in the complement of $R(x)$.

Then $x$ and $\tau_{b, a}(x)$ intersect only at a finite number of positive integers, and there must exist a $k>0$ such that for all $j \geq k$ one has

$$
x_{j} \geq x_{j-b}+a
$$

or, for all $j \geq k$ one has $x_{j} \leq x_{j-b}+a$.

In the first case one proves inductively that $x_{k+l b} \geq x_{k}+l a$, so that $\underline{p} \geq a / b$ which is impossible. The second case also leads to a contradiction.

Thus we have $\rho=\bar{\rho}$.

Note that we cannot exclude the possibility that the forward rotation number, which was shown to exist, is infinite.

LEMMA 8.4. If $R(x)$ contains two or more elements, then the map $\varphi$ has positive topological entropy.

Proof. Let $a / b<c / d$ be two different elements of $R(x)$, and assume that we actually have $(b, a) \in J(x)$ and $(d, c) \in J(x)$. In particular we have $b>0$ and $d>0$.

We shall construct a supersolution $\bar{y}$ whose backward rotation number is $a / b$, and whose forward rotation number is $c / d$.

Since $x$ and $\tau_{b, a}(x)$ intersect infinitely often at a positive integer there must exist a $j \geq 0$ such that

$$
\begin{gathered}
x_{j} \leq \tau_{b, a}(x)_{j}=x_{j-b}+a \\
x_{j+1}>\tau_{b, a}(x)_{j+1}=x_{j+1-b}+a
\end{gathered}
$$

holds. In other words there must exist a $j \geq 0$ such that $x_{i}-\tau_{b, a}(x)_{i}$ increases from negative to positive at $j$.

For $i \leq j$ we define $\bar{y}_{i}$ as follows:

$$
\begin{gathered}
\bar{y}_{j}=x_{j}, \quad \bar{y}_{j-1}=x_{j-1}, \ldots, \bar{y}_{j-b+1}=x_{j-b+1} \\
\bar{y}_{j-b}=x_{j}-a, \quad \bar{y}_{j-b-1}=x_{j-1}-a, \ldots, \bar{y}_{j-2 b+1}=x_{j-b+1}-a
\end{gathered}
$$

and in general for $t=0,1,2, \ldots, b-1$, and $s \geq 0$ :

$$
\bar{y}_{j-s b-t}=x_{j-t}-s a \text {. }
$$


Then we claim that for $i \leq j-1$ one has $\Delta\left(\bar{y}_{i-1}, \bar{y}_{i}, \bar{y}_{i+1}\right) \leq 0$. Since the sequence $\bar{y}_{i}$ is periodic we only have to verify this for $i=j-1, j-2, \ldots, j-b$. For $j \geq i \geq j-b+1$ one has $\bar{y}_{i}=x_{i}$, so that

$$
\Delta\left(\bar{y}_{i-1}, \bar{y}_{i}, \bar{y}_{i+1}\right)=\Delta\left(x_{i-1}, x_{i}, x_{i+1}\right)=0 \quad(j-1 \geq i \geq j-b+2) .
$$

At $i=j-b+1$ one has

$$
\begin{aligned}
\Delta\left(\bar{y}_{i-1}, \bar{y}_{i}, \bar{y}_{i+1}\right) & =\Delta\left(x_{j-b+2}, x_{j-b+1}, x_{j}-a\right) \\
& \leq \Delta\left(x_{j-b+2}, x_{j-b+1}, x_{j-b}\right)=0
\end{aligned}
$$

since $\Delta$ is increasing in its third argument, and $x_{j}-a \leq x_{j-b}$. A similar argument can be used to verify the case $i=j-b$.

It remains to define $\bar{y}_{i}$ for $i>j$. Since $x$ and $\tau_{d, c}(x)$ also intersect at an infinite number of positive integers there must be a $k \geq 0$ such that

$$
\begin{gathered}
x_{k} \geq x_{k-d}+c \\
x_{k+1}<x_{k-d+1}+c .
\end{gathered}
$$

holds. Moreover we can choose $k$ (much) larger than $j$. Given this $k$ we define $\bar{y}_{i}=x_{i}$ for $j \leq i \leq k$, for any $t=1,2, \ldots, d$ and $s=0,1,2, \ldots$ we put

$$
\bar{y}_{k+s \cdot d+1}=x_{k+1}+s a \text {. }
$$

As above one easily verifies that $\bar{y}$ is a supersolution. Furthermore $\bar{y}$ has a forward and backward rotation number equal to $c / d$ and $a / b$ respectively.

A similar construction can be used to construct a subsolution $\underline{y}$ with forward rotation number $a / b$ and backward rotation number $c / d$.

The sub- and supersolution, $y$ and $\bar{y}$, satisfy the hypothesis (6.1) so that Theorem 7.1 tells us that the map $\varphi$ has positive topological entropy. This completes the proof of Lemma 8.4.

Proof of Theorem 8.1. If $\varphi$ has zero topological entropy, then by Lemma 8.4, the set $R(x)$ has at most one element, for any given solution $x$ of (2.2). By Lemma 8.3 this sequence must have a forward rotation number.

Observe that the reversed sequence $\tilde{x}_{k}=x_{-k}$ satisfies the recurrence relation

$$
\tilde{\Delta}\left(\tilde{x}_{i-1}, \tilde{x}_{i}, \tilde{x}_{i+1}\right)=0 \text {, }
$$

where $\tilde{\Delta}(r, s, t)=\Delta(t, s, r)$. The map corresponding to this (monotone) recurrence relation is conjugate to the inverse of $\varphi$ and therefore also has zero topological entropy. Therefore the sequence $\tilde{x}$ has a forward rotation number, and the sequence $x$ has a backward rotation number.

Proof of Theorem 8.2. Let $x \in X$ be a sequence which is periodic of type $(p, q)$. Then for any $(m, n)$ the sequences $x$ and $\tau_{m, n}(x)$ either do not intersect, or they intersect infinitely often (since they are periodic with the same period).

The periodicity also implies that if $x$ and $\tau_{m, n}(x)$ intersect then $x$ and $\tau_{m+q, n+p}(x)$ do too. So if $(m, n) \in J(x)$, then $(m+q, n+p) \in J(x)$ and both $n / m$ and $(n+p) /(m+q)$ beong to $R(x)$. Since we are assuming that $\varphi$ has zero topological entropy we can apply Lemma 8.4 to conclude that $n / m=(n+p) /(m+q)$. But this implies that $n / m=p / q$. 
Our other assumption was that $\operatorname{gcd}(p, q)=1$. Therefore there is an integer $l \geq 1$ such that $(m, n)=(l q, l p)$. This cannot be true, however, since we then would have $\tau_{m, n}(x)=x$ so that they do not intersect.

We see that the assumption that $x$ and $\tau_{m, n}(x)$ intersect for some $(m, n)$ leads to a contradiction. So $x$ does not intersect any of its translates, which means that it is a Birkhoff sequence.

\section{Existence of Birkhoff sub- and supersolutions in general}

We return to the more general monotone recurrence relation (2.4). Given any $\omega \in R$ the equation (2.4) need not have a Birkhoff solution with rotation number $\omega$. Instead of this, we have the following.

THEOREM 9.1. For any $\omega \in R$ there is a Birkhoff sequence $x \in B$ such that for some $\lambda \in \mathbf{R}$

$$
\Delta\left(x_{k-l}, \ldots, x_{k+m}\right)=\lambda \quad(k \in \mathbf{Z})
$$

(where $\lambda$ does not depend on $k$ ), and such that $x$ has rotation number $\omega$. In particular, $x$ is either a subsolution or a supersolution.

Using this theorem we shall also prove the next result.

THEOREM 9.2. If (2.4) has a Birkhoff subsolution $x^{(0)}$ and a Birkhoff supersolution $x^{(1)}$ then it also has a Birkhoff solution $x$.

If $\omega_{j}$ is the rotation number of $x^{(j)}$ then the rotation number of $x, \omega$, can be chosen between $\omega_{0}$ and $\omega_{1}$ (i.e., $\omega_{0} \leq \omega \leq \omega_{1}$ or $\omega_{1} \leq \omega \leq \omega_{0}$, depending on how $\omega_{0}$ and $\omega_{1}$ are ordered).

Although there is a superficial resemblance between this theorem and Theorem 4.2 , they are really different. The difference is of course that we do not assume that the sub- and supersolution in the last theorem are in any way ordered.

We would also like to point out that, in the case of monotone twist maps, relation 9.1 reduces to

$$
Y\left(x_{k+1}, x_{k}\right)=\bar{Y}\left(x_{k-1}, x_{k}\right)+\lambda
$$

in the notation of $\S 2$. Therefore solutions of 9.1 correspond to orbits of the 'translated twist map' given by

$$
F_{\lambda}(x, y)=(f(x, y), g(x, y)+\lambda),
$$

(again we use the notation of $\S 2$ ).

These maps have been studied before, e.g. by Chenciner in [Ch, §6]. Our main motivation for studying (9.1), or the $F_{\lambda}$ 's is that they seem to be a natural oneparameter family of maps, or recurrence relations associated to $\Delta$. Moreover Theorem 9.1 leads to a convenient proof of Theorem 9.2.

We begin the proof of Theorem 9.1 with the following observation. If there is a dense set of $\omega$ 's in $\mathbf{R}$, for which the theorem holds then it is true for any real $\omega$. Indeed, suppose we can construct Birkhoff orbits $x^{(j)} \in B$ with rotation numbers $\omega^{(j)}$ and suppose the $\omega^{(j)}$ converge to some $\omega \in \mathbf{R}$. Then we may assume that the $x^{(j)}$ satisfy $0 \leq x_{0}^{(j)} \leq 1$, and by the compactness criterion of $\S 3$ (see 3.1) we can extract a convergent subsequence. The limit of this subsequence will be the desired 
Birkhoff orbit with rotation number $\omega$. (This trick is not without precedent, see [Ka1].)

The theorem is also true if it holds for any $\Delta$ which satisfies the following strict monotonicity condition

$$
\Delta=\Delta\left(x_{-1}, \ldots, x_{+m}\right) \text { is strictly monotone in each } x_{j} \text { except } x_{0} .
$$

To see why this is true, let $\Delta$ be any function which satisfies $\underline{a}, \underline{b}$ and $\underline{c}$ from $\S 2$. Then

$$
\Delta_{\varepsilon}\left(x_{-l} \cdots x_{+m}\right)=\Delta\left(x_{-l} \cdots x_{m}\right)+\varepsilon\left(\sum_{-l}^{m} x_{j}-(l+m+1) x_{0}\right)
$$

still satisfies $\underline{\mathrm{a}}, \underline{\mathrm{b}}$ and $\underline{\mathrm{c}}$, and in addition satisfies condition (9.2), for any $\varepsilon>0$. So for any $\varepsilon>0, \Delta_{\varepsilon}$ has a Birkhoff sequence $x^{\varepsilon}$ which satisfies $(9.1)$, and has rotation number $\omega$. Again we may take a cluster point of the $x^{\varepsilon}$ as $\varepsilon \downarrow 0$ to obtain a solution of (9.1) for our original $\Delta$ with the appropriate rotation number.

So from here on we assume that $\omega=p / q$, and that $\Delta$ satisfies (9.2). Moreover we suppose $\operatorname{gcd}(p, q)=1$, and $q \geq 4$.

We shall show that a solution of (9.1) must exist by means of a homotopy argument.

Let $B_{p, q}$ denote the set of $(p, q)$ periodic Birkhoff sequences. Then $B_{p, q}$ is not empty; it contains the sequence $x_{j}=j p / q$. The set $B_{p, q}$ is a closed convex subset of of the vectorspace $X$. If we let $X_{p, q}$ denote the affine subspace of $X$ which comprises all $(p, q)$-periodic sequences, then $B_{p, q}$ has nonempty interior in $X_{p, q}$.

By the compactness criterion of $\S 3$, the set

$$
D=\left\{x \in B_{p, q}: x_{0}=0\right\}
$$

is compact. In fact $D$ is homeomorphic to the $(q-1)$ dimensional unit ball in $\mathbf{R}^{q-1}$, and the mapping

$$
x \in B_{p, q} \rightarrow\left(x_{0}\right) \times\left(0, x_{1}-x_{0}, \ldots, x_{q}-x_{0}\right) \in \mathbf{R} \times D
$$

is a homeomorphism of $B_{p, q}$ with $\mathbf{R} \times D$.

The $\mathbf{Z}$-action on $B_{p, q}$ induces a $\mathbf{Z}$-action on $\mathbf{R} \times D$. This induced action is given by 'addition to the first coordinate', i.e. $(x, d) \cdot k \rightarrow(x+k, d)$ if $x \in \mathbf{R}$ and $d \in D$.

We let $K$ denote the quotient

$$
K=B_{p, q} / \mathbf{Z}
$$

and let $\partial K$ be its boundary $\partial K=\left(\partial B_{p, q}\right) / \mathbf{Z}$, where $\partial B_{p, q}$ is the boundary of $B_{p, q}$ in $X_{p, q}$.

Using the homeomorphism (9.3) we see that $K \cong S^{1} \times D$ and $\partial K \cong S^{1} \times S^{q-2}$.

On $K$ we define a continuous mapping into $\mathbf{R}^{4}$, given by

$$
\begin{aligned}
& \delta: K \rightarrow \mathbf{R}^{q} \\
& \delta_{i}(x)=\Delta\left(x_{i-l}, \ldots, x_{i+m}\right) \quad(1 \leq i \leq q) .
\end{aligned}
$$

At first sight it looks as if $\delta$ is only defined on $B_{p, q}$, but the periodicity of $\Delta$ makes that $\delta$ is also well defined on the quotient.

Given $\delta$ we define one more mapping, $\varepsilon$ :

$$
\varepsilon_{k}(x)=\delta_{k}(x)-\frac{1}{q} \sum_{j=1}^{q} \delta_{j}(x) \quad(k=1,2, \ldots, q) .
$$


Thus, $\varepsilon$ is a map from $K$ to the subspace

$$
\Lambda=\left\{\left(\delta_{1}, \ldots, \delta_{q}\right) \in \mathbf{R}^{q} \mid \delta_{1}+\delta_{2}+\cdots+\delta_{q}=0\right\}
$$

of $\mathbf{R}^{q}$. We have constructed $\varepsilon$ in such a manner that solutions of (9.1) are exactly the zeroes of $\varepsilon$.

LEMMA 9.2. $\varepsilon$ does not vanish on $\partial K$.

Proof Let $x$ and $y$ be any pair of solutions of (9.1), and assume that $x \leq y$. Then we claim that the strict monotonicity hypothesis (9.2) implies $x \ll y$.

Indeed, if for some $k$ we would have $x_{k}=y_{k}$ then

$$
\Delta\left(x_{k-l}, \ldots, x_{k+m}\right)=\Delta\left(y_{k-l}, \ldots, y_{k+m}\right)(=\lambda)
$$

combined with the strict monotonicity of $\Delta$ shows that we really have $x_{k-j}=y_{k-j}$ for $-l \leq j \leq+m$. By repeating this argument one finds that $x_{i}=y_{i}$, or $x_{i}<y_{i}$ holds for all $i \in Z$.

An $x \in X_{p, q}$ belongs to $B_{p, q}$ if and only if for any pair of integers $(k, l)$ one has $\tau_{k, l}(x) \geq x$ or $\tau_{k, l}(x) \leq x$. The same point will belong to the boundary of $B_{p, q}$ if, in addition, for some $(k, l)$ one has $\tau_{k, l}(x) \geq x$ (or $\left.\tau_{k, l}(x) \leq x\right)$ but not $\tau_{k, l}(x) \gg x$ (or $\tau_{k, I}(x) \ll x$ respectively).

If this point were a solution of $(9.1)$, then $\tau_{k, l}(x)$ would be one too so that $\tau_{k, l}(x) \geq x$ would imply $\tau_{k, l}(x) \gg x$. But that would contradict $x \in \partial B_{p, q}$.

We conclude that $\varepsilon$ does not vanish on the boundary of $K$.

This lemma shows that $\varepsilon$ defines a mapping from $\partial K$ into $\Lambda \backslash\{0\}$. We consider the associated homomorphism $\pi_{q-2}(\varepsilon): \pi_{q-2}(\partial K) \rightarrow \pi_{q-2}(\Lambda \backslash\{0\})$ of homotopygroups. Note that these groups are well defined since we have assumed that $q \geq 4$.

We already saw that $\partial K \cong S^{1} \times S^{q-2}$ so that

$$
\pi_{q-2}(\partial K) \cong \pi_{q-2}\left(S^{1}\right) \times \pi_{q-2}\left(S^{q-2}\right) \cong Z \text {. }
$$

Furthermore, $\Lambda \backslash\{0\}$ has the homotopy type of $S^{q-2}$ so that $\pi_{q-2}(\Lambda \backslash\{0\}) \cong \mathbf{Z}$. It follows that the homomorphism $\pi_{q-2}(\varepsilon)$ is represented by an integer, which we shall call its degree. This degree is defined up to its sign, which depends on the choice of generators of the groups $\pi_{q-2}(\partial K)$ and $\pi_{q-2}(\Lambda \backslash\{0\})$ which we have implicitly made.

If the degree of $\varepsilon$ is nonzero then $\varepsilon$ must vanish somewhere in $K$. Indeed, if $\varepsilon(x) \neq 0$ for all $x \in K$, then we may write $\left.\varepsilon\right|_{\partial K}=\varepsilon \circ j$, where $j: \partial K \rightarrow K$ is the inclusion mapping. Since $\pi_{q-2}(K) \cong \pi_{q-2}\left(S^{\prime} \times D\right)=0$ (here we use $q \geq 4$, and the convexity of $D$ ) the composition $\pi_{q-2}(\varepsilon) \cdot \pi_{q-2}(j)$ vanishes, so that the degree of $\varepsilon$ is zero. Therefore we can complete the proof by showing that this degree is nonzero.

With this end in mind we observe that the set

$$
\left\{\Delta \in C^{0}\left(\mathbf{R}^{1+m+1}\right) \mid \Delta \quad \text { satisfies } \underline{a}, \underline{b}, \underline{c} \text { of } \S 2 \text {, and also }(9.2)\right\}
$$

is convex and therefore certainly connected. This means that all the maps $\varepsilon$ which we have just considered are homotopic on $\partial K$, and they all have the same degree. We can compute this degree by looking at any particular example that pleases us. 
Consider the following choice of $\Delta$ :

$$
\Delta\left(x_{-l}, \ldots, x_{m}\right)=x_{-1}+\cdots+x_{m}-(l+m+1) \cdot x_{0} .
$$

We choose coordinates $\xi_{1}, \ldots, \xi_{q}$ on $X_{p, q}$ which are defined by

$$
x_{k}=k p / q+\xi_{k} \quad(1 \leq k \leq q) .
$$

For $k \leq 0$ or $k \geq q+1$ we define $\xi_{k}$ by assuming that $\xi_{k+q}=\xi_{k}$ holds. We may identify $X_{p, q}$ with the group ring of $Z / q Z$, so that multiplication on $X_{p, q}$ is defined by convolution of the $\xi_{k}$-coordinates:

$$
(\xi * \eta)_{k}=\sum_{j=0}^{q-1} \xi_{k-j} \eta_{j}
$$

The map $\delta: X_{p, q} \rightarrow \mathbf{R}^{q}$ is simply given by

$$
\delta(\xi)=\gamma * \xi+\frac{(l+m+1)(m-l)}{2} \frac{p}{q}
$$

where $\gamma=\sum_{-l}^{+m} e_{j}-(I+m+1) \cdot e_{0}$, and $e_{j}$ represents $j \in \mathbf{Z} / q \mathbf{Z}$ in the group ring of $\mathbf{Z} / \mathbf{q Z}$. The map $\varepsilon$ is given by

$$
\varepsilon(\xi)=\gamma * \xi-\operatorname{Av}(\gamma * \xi)=\gamma *(\xi-\operatorname{Av}(\xi))
$$

where $\operatorname{Av} \xi=\left(\xi_{1}+\cdots+\xi_{q}\right) / q$.

The characters of $\mathbf{Z} / q \mathbf{Z}$ are given by

$$
X_{j}\left(e_{k}\right)=e^{2 \pi i j k / q}
$$

for $j, k \in \mathbf{Z} / q \mathbf{Z}$.

One finds that

$$
X_{j}(\gamma)=\sum_{k=-1}^{+m} e^{2 \pi i j k / q}-(l+m+1)
$$

so that if $j \neq 0 \bmod (q)$ one has

$$
\left|X_{j}(\gamma)+l+m+1\right|<l+m+1
$$

and thus $\operatorname{Re}\left(X_{j}(\gamma)\right)<0$, and in particular one sees that $X_{j}(\gamma) \neq 0$.

This implies that the only zeros of $\varepsilon$ are given by $\xi_{k}=$ constant, i.e. by $x_{k}=(k p / q)+x_{0}$. This discussion also allows us to compute the degree of the map $\varepsilon$. Indeed, the map $\varepsilon$ is linear, and the kernel of its restriction to $\left\{x \in X_{p, q}: x_{0}=0\right\}$ consist of all constant $\xi$ with $\xi_{0}=0$; in other words, $\varepsilon$ restricted to $\left\{x \in X_{p, q}: x_{0}=0\right\}$ is injective. Hence the degree of $\varepsilon \mid \partial D \rightarrow \Lambda-\{0\}$ is \pm 1 . Since the inclusion of $\partial D$ in $\partial K \cong S^{1} \times \partial D$ induces an isomorphism on the $(q-2)$ dimensional homotopy groups the degree of $\varepsilon: \partial K \rightarrow \Lambda-\{0\}$ is also given by \pm 1 .

The proof of Theorem 9.1 is complete.

We turn to Theorem 9.2.

Define the following two subsets of $\mathbf{R}$.

$\operatorname{Rot}_{+}=\{\omega \in \mathbf{R} \mid \exists x \in B: \quad x$ is a supersolution and $x$ has rotation number $\omega\}$

$\operatorname{Rot}_{-}=\{\omega \in \mathbf{R} \mid \exists x \in B: \quad x$ is a subsolution and $x$ has rotation number $\omega\}$. 
It follows directly from Theorem 9.1 that the union of Rot R $_{+}$and Rot R $_{-}$is all of $\mathbf{R}$. Moreover if we define:

$$
\operatorname{Rot}_{0}=\{\omega \in \mathbf{R} \mid \text { there exists a Birkhoff solution of rotation number } \omega\}
$$

then we have $\operatorname{Rot}_{0}=\operatorname{Rot}_{+} \cap \operatorname{Rot}_{-}$. Indeed, the inclusions ' $\subset$ ' are trivial, and if $\omega$ belongs to Rot ${ }_{+}$and Rot. then there exists a Birkhoff subsolution $\underline{x}$ and a Birkhoff supersolution $\bar{x}$, both of which have rotation number $\omega$. For a suitable large integer $M$ one will have $\underline{x}-M<\bar{x}+M$ (in view of the inequalities (3.1)) and Theorem 4.2 plus the addendum following it tell us that there exists a Birkhoff solution between $\underline{x}-M$ and $\bar{x}+M$. Hence $\omega \in \operatorname{Rot}_{0}$.

Using the compactness property described in $\S 3$ one easily shows that the sets Rot $_{+}$and Rot $_{-}$are closed.

The hypothesis of Theorem 9.2 is that $\omega_{0} \in \operatorname{Rot}_{-}$and $\omega_{1} \in \operatorname{Rot}_{+}$. Assume that $\omega_{0} \leq \omega_{1}$. Then we have just shown that there are two closed sets $A_{ \pm}=\left[\omega_{0}, \omega_{1}\right] \cap \operatorname{Rot}_{ \pm}$ whose union is the interval $\left[\omega_{0}, \omega_{1}\right]$, and neither of which is empty. Since the interval is connected the intersection $\boldsymbol{A}_{+} \cap \boldsymbol{A}_{-}$is nonempty. Therefore there exists a Birkhoff orbit with rotation number $\omega \in\left[\omega_{0}, \omega_{1}\right]$. The same line of reasoning can be followed when $\omega_{1} \leq \omega_{0}$, so that we have completed the proof of Theorem 9.2.

If we are dealing with the two lowest dimensional cases $l=0, m=1$ (i.e. degree one circle maps) and $l=m=1$ (i.e. twist maps of a 2-dimensional annulus) then we can improve the previous results.

THEOREM $9.3(l=0, m=1)$. If $\varphi: S^{1} \rightarrow S^{1}$ has degree one then it has a Birkhoff orbit. Proof. Let $f: \mathbf{R} \rightarrow \mathbf{R}$ be a lift of $\varphi$, i.e. $f \in C^{0}(\mathbf{R}, \mathbf{R})$ and $f(x)+1=f(x+1)$. Then $M=\sup |f(x)-x|$ is finite, and one easily verifies that

$$
\underline{x}_{n}=-n \cdot M, \quad \bar{x}_{n}=+n \cdot M
$$

are a sub- and a supersolution for $\Delta\left(x_{0}, x_{1}\right)=x_{1}-f\left(x_{0}\right)$. By Theorem 9.2 we know that there exists a Birkhoff solution of $\Delta\left(x_{n}, x_{n+1}\right)=0$.

In the next Theorem we consider a monotone twist map $\varphi$ of the two dimensional annulus $S^{1} \times \mathbf{R}$.

THEOREM 9.4. If there exist $a<b$ such that $\varphi$ maps the ring $S^{1} \times[a, b]$ into itself then $\varphi$ has a Birkhoff orbit.

Proof. Let $F=(f, g)$ be lift of $\varphi$, and define the functions $Y, \bar{Y}$ and $\Delta$ as in the beginning of section two. In addition, we consider two auxiliary functions defined by $f_{a}(x)=f(x, a)$ and $f_{b}(x)=f(x, b)$.

Then $f_{a}$ represents a degree-one circle map, and the previous theorem says that there exists a Birkhoff sequence $\underline{x}_{n}$ for which $\underline{x}_{n+1}=f_{a}\left(\underline{x}_{n}\right)(n \in Z)$ holds. If one checks the definition of $Y, \bar{Y}$ and $\Delta$ then one finds:

$$
\begin{aligned}
& Y\left(\underline{x}_{n}, \underline{x}_{n+1}\right)=a \\
& \bar{Y}\left(\underline{x}_{n-1}, \underline{x}_{n}\right)=g\left(x_{n-1}, Y\left(x_{n-1}, x_{n}\right)\right) \geq a \\
\rightarrow & \Delta\left(\underline{x}_{n-1}, \underline{x}_{n}, \underline{x}_{n+1}\right) \geq 0 .
\end{aligned}
$$


So $\underline{x}_{n}$ is a Birkhoff subsolution. By a similar argument a Birkhoff orbit for the map $f_{b}$ must be a Birkhoff supersolution for $\Delta$.

It follows that the map $\varphi$ has a Birkhoff orbit.

10. The symplectic case

In this final section we draw the reader's attention to a subclass of monotone recurrence relations whose associated map preserves a symplectic structure. Not surprisingly, these recurrence relations are those which can be derived from a variational principle.

Our construction goes like this. Let $h \in C^{2}\left(\mathbf{R}^{d+1}\right)$ satisfy

$$
h_{x_{k} x_{l}} \geq \delta \quad(k \neq l)
$$

for some constant $\delta>0$. Then we define the formal sum $W(x)$ for $x \in X$ by

$$
W(x)=\sum_{j \in \mathbf{Z}} h\left(x_{j}, x_{j+1}, \ldots, x_{j+d}\right) .
$$

This sum will not converge in general, but if $x, y \in X$ coincide except for a finite number of components, then the a priori formal expression $W(x)-W(y)$ will lead to a finite sum. Hence one can define the derivative of $W$ at an $x \in X$. One finds that:

$$
\begin{aligned}
& \frac{\partial W}{\partial x_{j}}=h_{d}\left(x_{j-d}, \ldots, x_{j}\right)+h_{d-1}\left(x_{j-d+1}, \ldots, x_{j+1}\right)+\cdots+h_{0}\left(x_{j}, \ldots, x_{j+d}\right) \\
& \quad \stackrel{\text { def }}{=} \Delta\left(x_{j-d}, \ldots, x_{j+d}\right) .
\end{aligned}
$$

The requirement that $W$ be stationary at $x \in X$ is therefore equivalent to the recurrence relation

$$
\Delta\left(x_{j-d}, \ldots, x_{j+d}\right)=0 \quad(j \in \mathbf{Z})
$$

If we assume that our original 'generating function' $h$ is periodic in the sense that

$$
h\left(x_{0}+1, \ldots, x_{d}+1\right) \equiv h\left(x_{0}, \ldots, x_{d}\right)+\Phi
$$

holds for some constant $\Phi \in \mathbf{R}$ then $\Delta$ satisfies the periodicity requirement of $\S 2$. From

$$
\frac{\partial}{\partial x_{i}} \Delta\left(x_{-d}, \ldots, x_{+d}\right)=h_{d-i, d}\left(x_{i-d}, \ldots, x_{i}\right)+\cdots+h_{0, i}\left(x_{0}, \ldots, x_{d}\right)
$$

if $0<i \leq d$ and a similar expression for $\partial \Delta / \partial x_{i}$ if $-d \leq i<0$ one sees that $\Delta$ also satisfies the monotonicity condition, and the coerciveness condition of $\S 2$. We conclude that the recurrence relation (10.2) is monotone as we have defined the term.

The recurrence relation (10.2) therefore defines a $C^{1}$ diffeomorphism of the $2 d$-dimensional annulus $A^{2 d} \simeq S^{1} \times R^{2 d-1}$. We denote this map by $\varphi_{\Delta}$ (as in $\S 2$ ).

We proceed to construct an invariant symplectic form for $\varphi_{\Delta}$.

Consider the 'partial action' function

$$
S\left(x_{1}, \ldots, x_{d}, \bar{x}_{1}, \ldots, \bar{x}_{d}\right)=\sum_{j=1}^{d} h\left(x_{j} \cdots x_{d} \bar{x}_{1} \cdots \bar{x}_{j}\right) .
$$


If $x \in X$ is a solution of (10.2), then

$$
S_{1}=S\left(x_{-d+1}, \ldots, x_{0}, x_{1}, \ldots, x_{d}\right)+S\left(x_{1} \cdots x_{d}, x_{d+1}, \ldots, x_{2 d}\right)
$$

is stationary with respect to variations of $x_{1}, \ldots, x_{d}$. Regarding $S_{1}$ as a function of $x_{1}, \ldots, x_{d}$ the relation $d S_{1}=0$ may be written as

where

$$
\sum \bar{Y}_{j}\left(x_{-d+1}, \ldots, x_{d}\right) d x_{j}=\sum Y_{j}\left(x_{1}, \ldots, x_{2 d}\right) d x_{j},
$$

$$
\begin{aligned}
Y_{j}\left(x_{1}, \ldots, x_{d}, \bar{x}_{1}, \ldots, \bar{x}_{d}\right) & =\frac{\partial S}{\partial x_{j}} \quad(1 \leq j \leq d) \\
\bar{Y}_{j}\left(x_{1} \cdots x_{d}, \bar{x}_{1} \cdots \bar{x}_{d}\right) & =-\frac{\partial S}{\partial \bar{x}_{j}} \quad(1 \leq j \leq d) .
\end{aligned}
$$

It follows from (10.3) that, if we write $\theta$ for $Y_{1} d x_{1}+\cdots+Y_{d} d x_{d}$ then

$$
\theta-\varphi_{\Delta}^{*}(\theta)=d S
$$

so that $\varphi_{\Delta}$ preserves the two form $-d \theta=\omega$. The matrix whose $(i, j)$ entry is $\partial Y_{j} / \partial x_{i+d}$ is lower-triangular, with positive numbers on the diagonal, so that the 2-form $d \omega=-\left(d Y_{1} \wedge d x_{1}+\cdots+d Y_{d} \wedge d x_{d}\right)$ has maximal rank, and is a symplectic form.

A very special case arises if one takes

$$
h\left(x_{0}, \ldots, x_{d}\right)=-\frac{1}{2} \sum_{j=1}^{d} \gamma_{j}\left(x_{0}-x_{j}\right)^{2}+\frac{k}{2 \pi} \cos 2 \pi x_{0}
$$

with $\gamma_{1}, \ldots, \gamma_{d}>0$ and $k \in \mathbf{R}$ constants. The assoicated $\Delta$ is

$$
\Delta\left(x_{-d}, \ldots, x_{d}\right)=\sum_{j=1}^{d} \gamma_{j}\left(x_{-j}-2 x_{0}+x_{+j}\right)+k \sin \left(2 \pi x_{0}\right)
$$

and the corresponding map $\varphi_{\Delta}$ generalizes the so called 'standard map' (i.e. the case $d=1, \gamma_{1}=1$ ).

As an application of the results of $\S 7$ we note that when

$$
k \geq \sum_{j=1}^{d} j \gamma_{j}
$$

one has a supersolution $\bar{x}_{j}=\frac{3}{4}+|j|$ and a subsolution $\underline{x}_{j}=\frac{1}{4}-|j|$ which satisfy (6.1), so that the map $\varphi_{\Delta}$ has positive entropy.

By analogy with the Aubry-Le Daeron theory [ALD] one could say that the solutions of (10.2) describe the equilibrium states of a bi-infinite chain of particles in which any sequence of $d+1$ consecutive particles contributes an amount equal to $-h\left(x_{j}, \ldots, x_{j+d}\right)$ to the total energy of the chain. The number $x_{j}$ then represents the position of the $j$ th particle. See figure 3. From here on we assume that $h$ is truly periodic, i.e. that $\Phi=0$.

As in the Aubry-Le Daeron theory one can show that the function

$$
W(x)=\sum_{i=1}^{q} h\left(x_{i}, \ldots, x_{i+d}\right)
$$




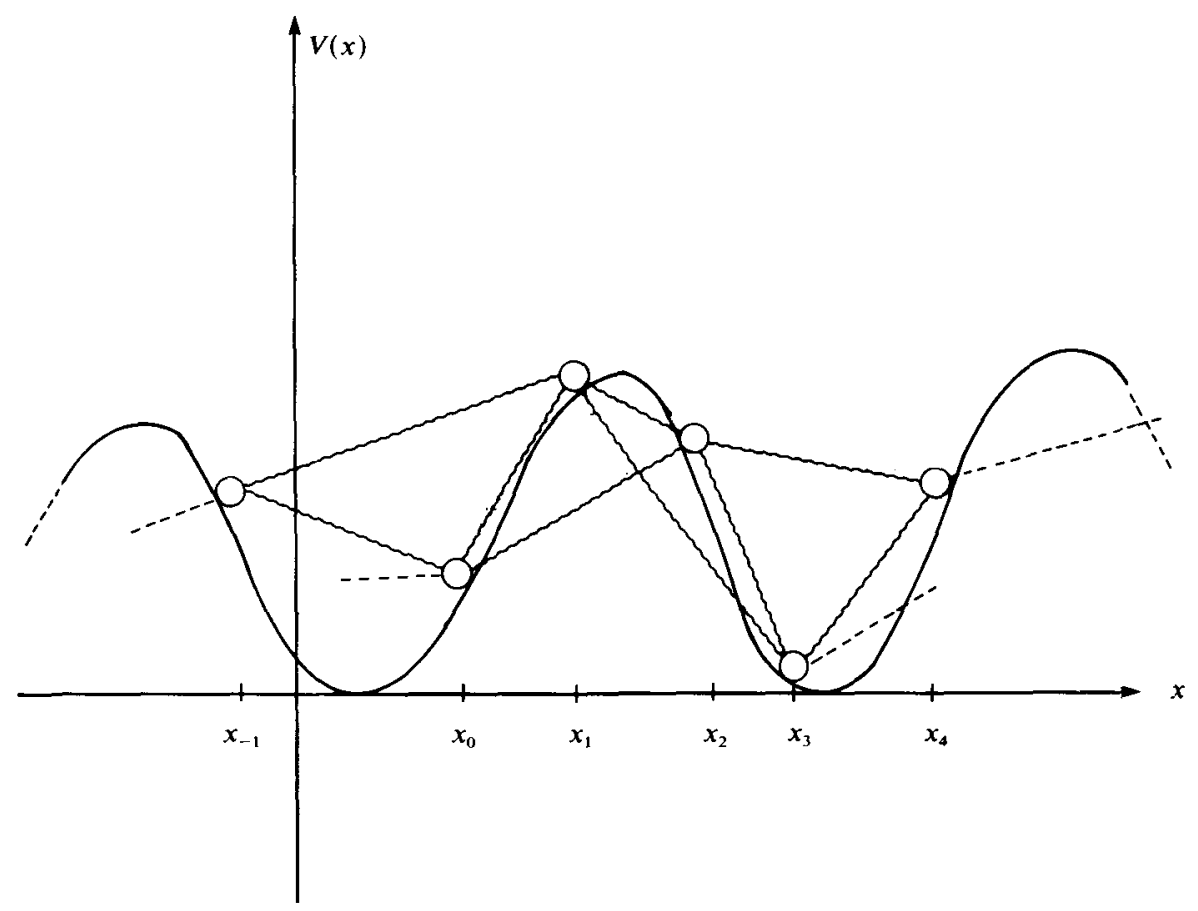

FIGURE 3. A chain of particles with nearest and second nearest neighbour interaction.

is well defined on $X_{p, q} / Z$, and in fact is proper on this space. Hence $W$ achieves its maximum on $X_{p, q}$, and for any $(p, q)$ we obtain the existence of a periodic orbit of $\varphi_{\Delta}$ of type $(p, q)$.

Using the positivity of the mixed derivatives one also shows that for $x, y \in X_{p, q}$ one has

$$
W(x)+W(y) \leq W(x \vee y)+W(x \wedge y)
$$

where $\vee$ and $\wedge$ denote the usual lattice operations on $X_{p, q}$ (one could adapt the proof on p. 520 of [Ma1]). Thus if $x$ maximizes $W$ on $X_{p, q}$ then so does $\tau_{k, l} x$ for any $k, l$ and one finds that

$$
2 W(x) \leq W\left(x \vee \tau_{k, l}(x)\right)+W\left(x \wedge \tau_{k, l}(x)\right) \leq 2 W(x) .
$$

Hence $x \vee \tau_{k, l}(x)$ and $x \wedge \tau_{k, l}(x)$ also maximize $W$, and therefore must be solutions of (10.2). Since our $\Delta$ satisfies condition (9.2) the proof of Lemma 9.2 indicates that we either have $x \vee \tau_{k, l}(x) \gg x$ or $x \vee \tau_{k, l}(x)=x$; in other words $x$ and $\tau_{k, l}(x)$ are ordered.

The conclusion is that any $x \in X_{p, q}$ which maximizes $W$ is a Birkhoff sequence. Finally we note that, just as in the Aubry-Le Daeron case, one can obtain Birkhoff orbits with prescribed rotation number by taking limits of similar orbits with rational rotation number. 


\section{REFERENCES}

[A] S. B. Angenent. The periodic orbits of an area preserving twistmap. Comm. Math. Phys. 115 (1988), 353-374.

[ALD] S. Aubry \& P. Y. Le Daeron. The discrete Frenkel-Kontorova model and its generalizations. Physica 8D (1983), 381-422.

[Ba] V. Bangert. Mather sets for twist maps and geodesics on tori. To appear in Dynamics Reported, Vol. 1.

[Bo] P. Boyland. Braid types and a topological method of proving positive entropy. Preprint.

[Ch] A. Chenciner. Bifurcations de points fixes elliptiques II, orbites periodiques et ensembles de Cantor invariantes. Inventiones. Math. 80 (1985), 81-106.

[Ha1] G. R. Hall. A topological version of a theorem of Mather on twist maps. Ergod. Th. \& Dynam. Sys. 4 (1984), 585-603.

[Ha2] G. R. Hall. A topological version of a theorem of Mather's on shadowing in monotone twist maps. Preprint.

[Ka1] A. Katok. Some remarks on Birkhoff and Mather twist theorems. Ergod. Th. \& Dynam. Sys. 2 (1982), 185-194.

[Ka2] A. Katok. Lyapunov exponents, entropy and periodic orbits for diffeomorphisms. Publ. Math. de I'I.H.E.S. 51 (1980), 137-174.

[Ma1] J. N. Mather. More Denjoy minimal sets for area-reserving diffeomorphisms. Comm. Math. Helv. 60 (1985), 508-557.

[Ma2] J. N. Mather. Dynamics of area preserving mappings. Preprint.

[Wa] P. Walters. An introduction to ergodic theory. Grad. Texts in Math., Springer-Verlag: New York, 1982. 